\title{
Heat generated during electrochemical double-layer capacitor "self-discharge"
}

\author{
Andrzej Lewandowski • Pawel Jakobczyk • \\ Martyna Gnat $\cdot$ Ewelina Rudnicka
}

Received: 31 October 2013/Accepted: 10 January 2014/Published online: 24 January 2014

(C) The Author(s) 2014. This article is published with open access at Springerlink.com

\begin{abstract}
Eight commercial 10F electrochemical doublelayer capacitors (EDLCs) were connected together and placed in a container filled with mineral oil. The whole system was placed into a Dewar container. Temperature variation and heat exchanged between the test EDLC and the environment during its charging, discharging, and "self-discharge" were measured, together with voltage $U$ changes. Charge separation during charging was equivalent to a transition into a more ordered system, which results in entropy decrease, while discharging caused entropy increase (the Peltier-Seebeck effect). Consequently, a number of charging/discharging cycles led to a corresponding series of entropy and temperature changes. The final shape of temperature versus time curve during charging/discharging cycles was due to overlapping of irreversible Joule-Lenz and reversible Peltier heats. When charged EDLC was kept under the open-circuit condition, measured heat flow was negligible in comparison to energy loss calculated from potential drop, assuming that energy $E$ accumulated is proportional at any time to voltage to the second power (i.e., $E \sim U^{2}$ ). The result was interpreted assuming that the EDLC "self-discharge" phenomenon is not associated with energy loss by the device, but rather with charge redistribution between EDLC particles characterized by different time constants.
\end{abstract}

Keywords Electrochemical double-layer capacitor . Self-discharge $\cdot$ Heat exchange

A. Lewandowski $(\bowtie) \cdot$ P. Jakobczyk · M. Gnat · E. Rudnicka

Faculty of Chemical Technology, Poznan University of

Technology, 60965 Poznan, Poland

e-mail: Andrzej.Lewandowski@put.poznan.pl

\section{Introduction}

The energy $E$ accumulated by an electrochemical doublelayer capacitor (EDLC) is usually calculated according to Eq. (1) valid for dielectric and electrolytic capacitors:

$E=\frac{1}{2} C U^{2}$,

where $C$ is the capacitance and $U$ is potential difference between electrodes (voltage). A spontaneous voltage drop between EDLC electrodes, when it is kept under the opencircuit condition, is commonly called "self-discharge" and interpreted, according to Eq. (1), as a relatively fast energy loss $[1,2]$. The law of energy preservation suggests that the voltage drop can generate energy exchange with the environment. Recent papers describe a model of charge redistribution in porous electrodes as the reason for EDLC voltage changes [3-5]. The model does not assume energy dissipation, in contrast to leakage current and faradaic mechanisms. In addition, it has been recently shown that during EDLC operation, equations valid for dielectric and electrolytic capacitors do not hold in the case of EDLCs $[6,7]$. Therefore, the assumption that the energetic state of the EDLC is proportional at any time to the voltage to the second power may not be valid. All these indicate that voltage changes called "self-discharge" may not reflect the energetic state of the device. If voltage drop is due to energy loss, then it can be converted inside EDLC into reagents free energy (faradaic mechanism) or exchanged with the environment in the form of work, electromagnetic radiation, or heat. To our knowledge, there are no reports about the contribution of faradaic processes or electromagnetic radiation to EDLC fast voltage drop. Moreover, EDLCs cannot work under the open-circuit condition. Consequently, heat exchange seems to be the main process 
responsible for the "self-discharge" understood as energy loss. Heat generation during EDLC charging and discharging was observed [8], while the corresponding effect during its storage has not been reported under open-circuit conditions. The general aim of this study was to measure experimentally the level of heat exchange between the environment and an EDLC kept under the open-circuit condition, in comparison to that assumed from the "selfdischarge" curve (according to the Eq. (1)).

\section{Experimental}

10F commercial capacitors (PC10) were obtained from Maxwell Technologies. Each cell dimension was $29.6 \mathrm{~mm} \times$ $23.6 \mathrm{~mm} \times 3.5 \mathrm{~mm}$, surface area: $17.70 \mathrm{~cm}^{2}$ with a mass of $6.6 \mathrm{~g}$. Two sets of four PC10 EDLCs combined in parallel (with capacitance of $40 \mathrm{~F}$ ) were connected in a series (eight PC10 devices, with total nominal capacitance of $20 \mathrm{~F}$ ). The total surface and mass of eight EDLCs were $141.60 \mathrm{~cm}^{2}$ and $52.8 \mathrm{~g}$. Ultracapacitors were placed in a polypropylene container filled with $80.0 \mathrm{~g}$ of mineral oil (Finavestan A360B, Total Oil Australia Ltd.). High-resistivity Kanthal-D wire (Kanthal, $\mathrm{Fe}-\mathrm{Cr}-\mathrm{Al}$ alloy, $25.3 \mathrm{~cm}$ long, diameter $0.16 \mathrm{~mm}$, resistance $67.1 \Omega \mathrm{m}^{-1}$ ), was placed in the system (132.8 $\mathrm{g}$ of eight capacitors and oil). Temperature was measured with an NTC chip TT2-10KC3-10 thermistor (Tewa, Poland). In the narrow temperature range of 293-298 K, the $R=f(T)$ characteristic was linear with the temperature coefficient of $499 \Omega$ $\mathrm{K}^{-1}$. The whole system was placed into a Dewar container. Charging of capacitors and calibration of the system with the resistive wire was performed with the Atlas-Sollich 0461MBI electrochemical system. Resistance of the thermistor was measured with an ME-32 universal meter (Metex, Korea) with an accuracy of $10 \Omega$ (which is equivalent to $\pm 0.02 \mathrm{~K}$ ). Impedance spectrum was taken with the use of a G750 frequency response analyzer (Gamry, USA) at a frequency range of $100 \mathrm{kHz}-10 \mathrm{mHz}$, at the open-circuit potential and amplitude of $10 \mathrm{mV}$.

\section{Results and discussion}

Heat capacity of the system (capacitors, mineral oil, the thermistor, resistive wire, and the container) was determined from the calibration curve shown in Fig. 1. A current $I=0.12$ A flowing through the wire of resistance $R=17.14 \Omega$ during time of $t=600 \mathrm{~s}$ generated the Joule-Lenz heat of $Q_{\text {cal }}=$ $R I^{2} t=148.1 \mathrm{~J}$. The calibration procedure was performed three times. Heat capacity of the system detected from the slope of calibration curves was $c_{\mathrm{p}}=1.43 \pm 0.05 \mathrm{~J} \mathrm{~g}^{-1} \mathrm{~K}^{-1}$. Then the EDLC was charged from 0 to $5 \mathrm{~V}$ with a current of 2 A (within ca. 47 s) hence, accumulated energy, calculated

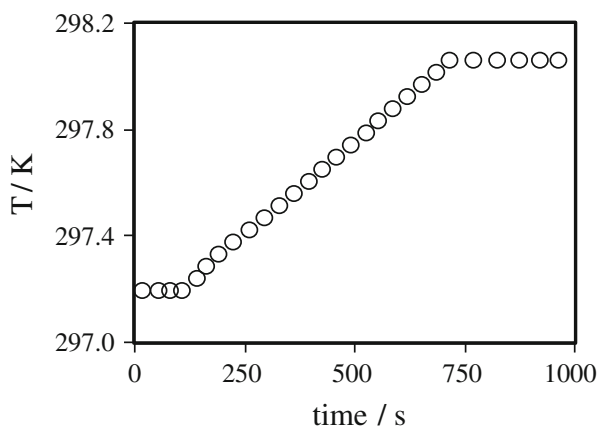

Fig. 1 Calibration curve of the system (the capacitor, mineral oil, thermistor, resistive wire, and the container, together $132.8 \mathrm{~g}$ ), placed in a Dewar. A current $I=0.12$ A flowing through the wire of resistance $R=17.14 \Omega$ during time of $t=600 \mathrm{~s}$ generated the JouleLenz heat, which changed the temperature. Heat capacity of the system $\left(c_{\mathrm{p}}=1.43 \pm 0.05 \mathrm{~J} \mathrm{~g}^{-1} \mathrm{~K}^{-1}\right)$ was detected from the slope of the calibration curve
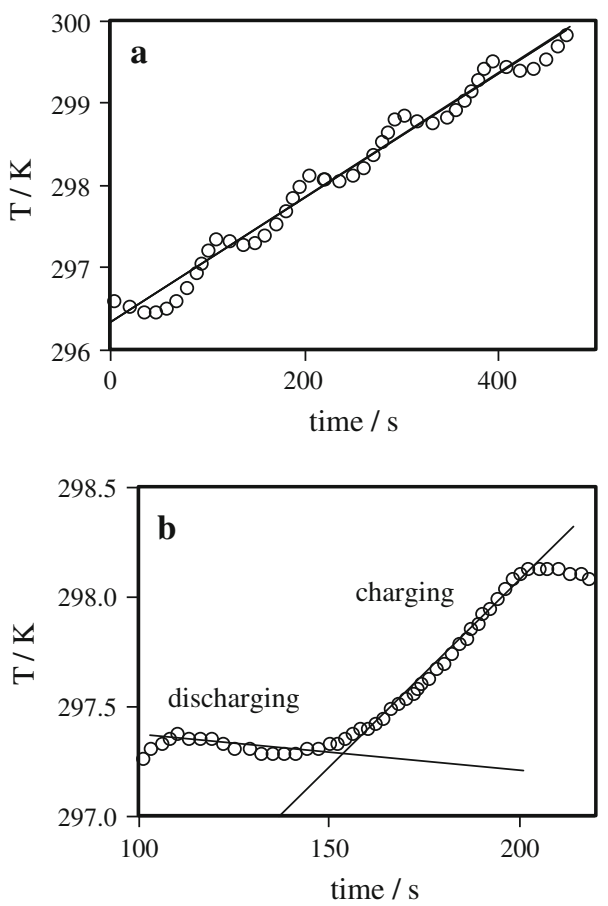

Fig. 2 Temperature changes detected during EDLC galvanostatic charging and discharging with a current of $2 \mathrm{~A}$ : a overlapping of the reversible Peltier-Seebeck and irreversible Joule-Lenz effects and b reversible Peltier-Seebeck effect

from Eq. (1), was $250 \mathrm{~J}$. Heat generated during the EDLC charging and discharging was not linear but had a shape shown in Fig. 2a. This is due to the fact that electric charge separation (charging) is equivalent to a transition into a more ordered system, which results in entropy decrease, while discharging causes entropy increase (the Peltier-Seebeck effect, Fig. 2b). Consequently, a number of charging/discharging cycles lead 

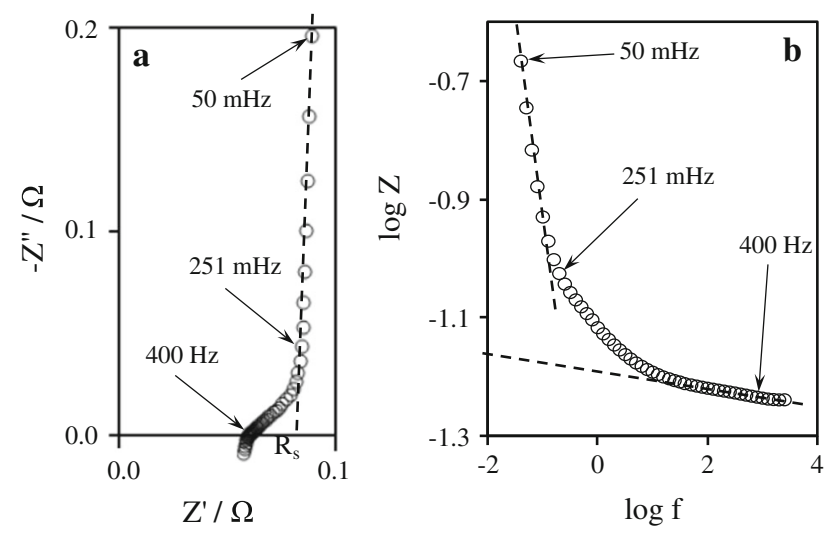

Fig. 3 Electrochemical impedance spectroscopy of the EDLC under study: a Nyquist plot and b Bode plot

to a corresponding series of entropy and temperature changes. The final shape of the $T=f(t)$ curve, shown in Fig. 2a, is due to overlapping of irreversible Joule-Lenz and reversible Peltier heats. The temperature recorded at $t=46 \mathrm{~s}$ was $T=296.48 \mathrm{~K}$, while the corresponding value for $t=425 \mathrm{~s}$ was $T=299.44 \mathrm{~K}$. Therefore, the temperature change with time was $\Delta T / \Delta t=0.36 \mathrm{~K} 47 \mathrm{~s}^{-1}$. The Joule-Lenz heat produced during one charging or discharging cycle $(47 \mathrm{~s})$ calculated from series resistance $R_{\mathrm{s}}=0.080 \Omega$ (measured with EIS, Fig. 3) was ca. $Q_{\mathrm{R}_{\mathrm{s}}}=R_{\mathrm{s}}{ }^{2} t=0.08 \Omega \times 4 \mathrm{~A}^{2} \times$ $47 \mathrm{~s}=15.04 \mathrm{~J}(P=0.32 \mathrm{~W})$. The corresponding value estimated from temperature changes (Fig. 2) was $Q_{\mathrm{J}-\mathrm{L}}=m c_{\mathrm{p}}$ $\Delta T=132.8 \mathrm{~g} \times 1.43 \mathrm{~J} \mathrm{~g}^{-1} \mathrm{~K}^{-1} \times 0.36 \mathrm{~K}=68.4 \mathrm{~J}\left(P_{\mathrm{J}-\mathrm{L}}=\right.$ $1.46 \mathrm{~W}$ ). The difference in both heats (or Joule-Lenz power) is due to the fact that, in addition to the series resistance, the corresponding resistance of the electrolyte in the pores should also be taken into account [7]. The Peltier heat may be calculated from the heat difference between discharging $\left(Q_{\text {Pelt }}^{\text {ch }}\right)$ and charging $\left(Q_{\text {Pelt }}^{\text {disch }}\right)$ cycles:

$Q^{\text {ch }}=Q_{\mathrm{J}-\mathrm{L}}+Q_{\text {Pelt }} \quad$ and $\quad Q^{\text {disch }}=Q_{\mathrm{J}-\mathrm{L}}-Q_{\text {Pelt }}$.

Slopes of the discharging and charging curves shown in Fig. $2 \mathrm{~b}$ were -0.0016 and $0.0171 \mathrm{~K} \mathrm{~s}^{-1}$, respectively. This leads to the Peltier heat (referred to each $47 \mathrm{~s}$ charging or discharging step) of $Q_{\text {Pelt }}=83.50 \mathrm{~J}\left(P_{\text {Pelt }}=1.78 \mathrm{~W}\right)$.

Figure 4 shows a typical $U=f(t)$ "self-discharge" curve of the EDLC under study after switching it off into the open-circuit condition after galvanostatic charging. At the beginning, $U$ decreases from its initial value $U_{\mathrm{o}}^{2}$ relatively fast and slows down with time. At the same time, temperature changes were measured with the thermistor. Measurements of potential and temperature changes were performed 10 times. Heat flow detected from temperature changes was calculated as $Q_{\text {flow }}=\mathrm{m} c_{\mathrm{p}} \Delta T$. This value may

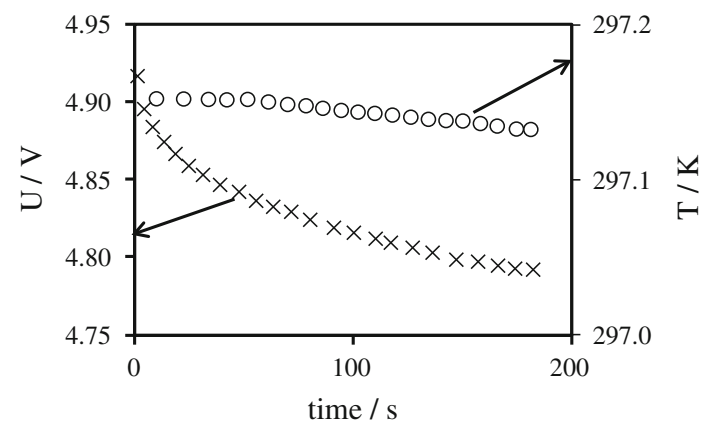

Fig. 4 The EDLC: (times symbols) "self-discharge" curve (spontaneous voltage changes under open-circuit conditions as a function of time) and (circles) temperature changes measured at the same time

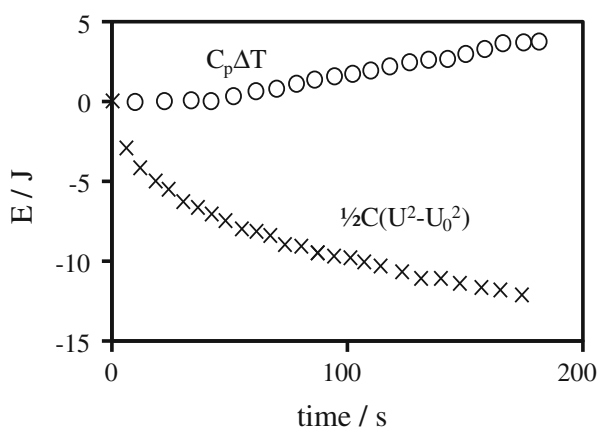

Fig. 5 Energy $E$ exchanged by the EDLC calculated from changes of (times symbols) voltage: $E=C / 2\left(U^{2}-U_{\mathrm{o}}^{2}\right)$ or (circles) temperature: $E=c_{\mathrm{p}} \Delta T$

be compared to the potential heat calculated from Eq. (1), assuming that during the potential decay, energy $E$ is exchanged exclusively in the form of heat. In other words, temperature and voltage changes were converted to the same (energy) units. If the temperature change reflected energy loss due to voltage drop, then both $c_{\mathrm{p}} \Delta T=f(t)$ and $1 / 2 C\left(U^{2}\right.$ $\left.-U_{\mathrm{o}}^{2}\right)=f(t)$ values should be present at the same curve. However, experimental data (an example shown in Fig. 5) did not support this assumption. It can be seen in Fig. 5 that energy measured from temperature changes during the "selfdischarge" was approximately constant (ca.: $4 \mathrm{~J}$ during $180 \mathrm{~s} \approx 1 \mathrm{~J}$ during $47 \mathrm{~s}$ ). However, the corresponding value calculated from voltage changes via Eq. (1) was much higher (ca. $13 \mathrm{~J}$ during $180 \mathrm{~s} \approx 3.39 \mathrm{~J}$ during $47 \mathrm{~s}$ ). Such a result was obtained in all 10 experiments (different charging current and final voltage). Therefore, the spontaneous voltage drop between EDLC electrodes, when it is kept under the open-circuit condition, commonly called the "self-discharge," cannot be interpreted as a relatively fast energy. However, the model of charge redistribution between EDLC particles characterized by different time constants can be applied to explain this phenomenon. 


\section{Conclusions}

When an EDLC is kept under the open-circuit condition, measured heat flow is negligible in comparison to energy loss calculated from potential drop, assuming that energy accumulated is proportional at any time to voltage to the second power. The result suggests that the EDLC "selfdischarge" phenomenon is not associated with energy loss by the device.

Acknowledgments Support of Grant 31-254/13 DS PB is gratefully acknowledged.

Open Access This article is distributed under the terms of the Creative Commons Attribution License which permits any use, distribution, and reproduction in any medium, provided the original author(s) and the source are credited.

\section{References}

1. Diab Y, Venet P, Gualous H, Rojat G (2009) Self-discharge characterization and modeling of electrochemical capacitor used for power electronics applications. IEEE Trans Power Electron 24:510-517

2. Wang G, Zhang L, Zhang J (2012) A review of electrode materials for electrochemical supercapacitors. Chem Soc Rev 41:797-828

3. Black J, Andreas HA (2009) Effects of charge redistribution on self-discharge of electrochemical capacitors. Electrochim Acta 54: 3568-3574

4. Kaus M, Kowal J, Sauer DU (2010) Modelling the effects of charge redistribution during self-discharge of supercapacitors. Electrochim Acta 55:7516-7523

5. Kowal J, Avaroglu E, Chamekh F, Senfelds A, Thien T, Wijaya D, Sauer DU (2011) Detailed analysis of the self-discharge of supercapacitors. J Power Sources 196:573-579

6. Lewandowski A, Jakobczyk P, Galinski M (2012) Capacitance of electrochemical double layer capacitors. Electrochim Acta 86: 225-231

7. Lewandowski A, Jakobczyk P, Galinski M, Biegun M (2013) Selfdischarge of electrochemical double layer capacitors. Phys Chem Chem Phys 15:8692-8699

8. Shiffer J, Linzen D, Sauer DU (2006) Heat generation in double layer capacitors. J Power Sources 160:765-772 